Judith Christina Eisele Inga-Marie Schaefer Jens Randel Nyengaard Heiner Post David Liebetanz Annemarie Brüel Christian Mühlfeld

\section{Effect of voluntary exercise on number and volume of cardiomyocytes and their mitochondria in the mouse left ventricle}

Received: 13 December 2006

Returned for 1. revision: 12 January 2007

1. Revision received: 17 January 2007

Returned for 2. revision: 12 February 2007

2. Revision received: 27 September 2007

Accepted: 1 October 2007

Published online: 14 November 2007

J.C. Eisele · I.-M. Schaefer · C. Mühlfeld

Dept. of Anatomy

University of Göttingen

Göttingen, Germany

J. Randel Nyengaard · C. Mühlfeld Stereology and Electron Microscopy

Research Laboratory

University of Århus

Arhus, Denmark

H. Post

Dept. of Cardiology and Pulmonary

Medicine

University of Göttingen

Göttingen, Germany

D. Liebetanz

Dept. of Clinical Neurophysiology

University of Göttingen

Göttingen, Germany

A. Brüel

Institute of Anatomy

University of Arhus

Arhus, Denmark

C. Mühlfeld

Institute of Anatomy, Division of Histology

University of Bern

Bern, Switzerland

Dr. C. Mühlfeld ( $\square)$

Universität Bern, Institut für Anatomie

Abteilung Histologie

Baltzerstraße 2

3000 Bern 9, Schweiz

$\because \quad$ Tel.: +41-31/631-8446

O Fax: +41-31/631-3807

苑 E-Mail: muehlfeld@ana.unibe.ch
Abstract Voluntary exercise (VE) has a beneficial influence on the heart and mean lifespan. The present study evaluates structural adaptations of cardiomyocytes and their mitochondria due to VE by new, unbiased stereological methods. Female, 7-9-week-old mice were randomly assigned to a control (CG, $n=7$ ) or VE group (EG, $n=7$ ). EG animals were housed in cages with free access to a running wheel and had a mean running distance of $6.7(1.8) \mathrm{km}$ per day. After 4 weeks, the hearts of all mice were processed for light and electron microscopy. We estimated the number and volume of cardiomyocytes by the disector method and the number and volume of mitochondria by estimation of the Euler number. In comparison to CG, VE did not have an effect on the myocardial volume of the left ventricle (CG: 93 (10), EG: $\left.103(17)\left(\mathrm{mm}^{3}\right)\right)$, the number of cardiomyocytes (CG: $2.81(0.27)$, EG: $\left.2.82(0.43)\left(\times 10^{6}\right)\right)$ and their number-weighted mean volume. However, the composition of the cardiomyocytes changed due to VE. The total volume of mitochondria (CG: $21.8(4.9)$, EG: $32.2(4.3)\left(\mathrm{mm}^{3}\right)$, $P<0.01)$ and the total number (CG: $3.76(0.44)$, EG: $7.02(1.13)\left(\times 10^{10}\right)$, $P<0.001)$ were significantly higher in EG than in CG. The mean numberweighted mitochondrial volume was smaller in EG than in CG $(P<0.05)$. In summary, VE does not alter ventricular volume nor cardiomyocyte volume or number but the oxidative capacity of cardiomyocytes by an increased mitochondrial number and total volume in the left ventricle. These structural changes may participate in the beneficial effects of VE.

Key words stereology - myocardium - Euler number - hypertrophy electron microscopy 


\section{Introduction}

Physical exercise increases the energy consumption of the whole organism, which requires the structural and functional capacity of the heart to meet the higher demands [33]. Depending on the degree (voluntary vs. strenuous) and the mode (weight vs. endurance training) of exercise the heart undergoes structural changes, which have adaptational character but-if pronounced-may be harmful [12, 26]. Myocardial changes induced by chronic exercise were subject to several both qualitative and morphometric light and electron microscopic studies. Bozner and Meessen [6] showed an increase in myocyte mitochondrial mass and in the mitochondria-to-myofibril ratio due to swimming exercise in the rat hearts using planimetry. Similar results were also obtained by Arcos et al. [5]. In the 1980s, Anversa's group performed several morphometric studies on physiological myocardial hypertrophy during development [2] and exercise [3] as well as pathological hypertrophy induced, for example, by myocardial infarction [4]. In their studies on rats trained by running exercise [3], they only found minor changes in left ventricular but larger changes in right ventricular volume. In contrast to the above-mentioned studies these authors did not find alterations of the mitochondria-to-myofibril ratio in the right ventricular rat myocardium. Similarly, Mattfeldt et al. [27] did not find changes in the volume fractions of myocardial components and cardiomyocyte organelles due to running exercise. Interestingly, however, Reichmann et al. [35] clearly showed that chronic electrical stimulation increased the number of mitochondrial profiles, the volume fraction of mitochondria and decreased the myofibril volume fraction in skeletal muscle. In hypertensive rats, which usually exhibit a decreased myocardial mitochondria-tomyofibril ratio, chronic exercise reversed the pathologically reduced oxidative capacity [9]. Differences among the cited investigations may be explained by the different degrees and modes of exercise applied to the animals and by the different morphometric approaches. Especially, the use of ratios may lead to false conclusions due to the reference trap and should therefore be regarded as obsolete [7, 30].

In recent years, there has been growing evidence that voluntary exercise (VE) where animals have free access to running wheels is an interesting alternative to forced exercise. It has been shown that VE increases mean lifespan $[15,16]$ and reduces the hydrogen peroxide production by myocardial mitochondria [20], with the latter probably delaying the ageing-associated loss of cardiomyocytes [37] and possibly being involved in the control of metabolic coronary flow regulation [10]. Furthermore, it has been shown that exercise prevented ischemia-associated arrhythmias in dogs [38]. However, in contrast to the above-mentioned studies on forced exercise, information about quantitative morphological changes of the myocardium due to VE is rare. Controversial reports exist whether voluntary wheel running increases heart mass $[1,32]$, however, an increase in subendocardial myocyte size has been reported [32].

Therefore, the present study evaluates the effects of voluntary wheel running on left ventricular myocardium using unbiased stereological estimations of number and volume of cardiomyocytes and their mitochondria. We found that neither volume nor number of cardiomyocytes as assessed by the method of Brüel and Nyengaard [8] were changed by VE. However, the total volume of mitochondria in the left ventricle was significantly increased by training. In order to evaluate whether the individual volume or the number of mitochondria caused the increase in total mitochondrial volume we estimated the number and the number-weighted mean volume of mitochondria in the left ventricle using the Euler-Poincaré characteristic [24]. Our results show a highly significant increase in mitochondrial number and a slight decrease in mean mitochondrial volume. The method for estimating the number of mitochondria by their connectivity was originally established for mitochondria of exocrine pancreas cells but has not been used to establish mitochondrial number and volume since its original description. Therefore, we hope that this study-apart from its biological significance-sets a new methodological standard for the quantitative morphological evaluation of myocardial structural changes due to higher metabolic demands.

\section{Material and methods}

\section{Animals}

Eighteen 7-9-week-old mass-matched female mice (Mus musculus, C57BL6J mice purchased from Charles River) were randomly assigned to a control group (CG) or to an exercise group (EG). For the morphological study, seven animals per group were chosen randomly. All mice were housed in individual cages with free access to standard food containing phytoestrogens and water. Over a period of 4 weeks, EG mice had free access to a running wheel within their cages to allow for VE training. Running distance and time were recorded continuously (Figs. 1, 2). Both CG and EG animals were housed in a room at $20-22^{\circ} \mathrm{C}$ with a $12 \mathrm{~h}: 12 \mathrm{~h}$ light:dark cycle.

Euthanization of the mice was reported to the Bioethical Committee of the District of Braunschweig, 


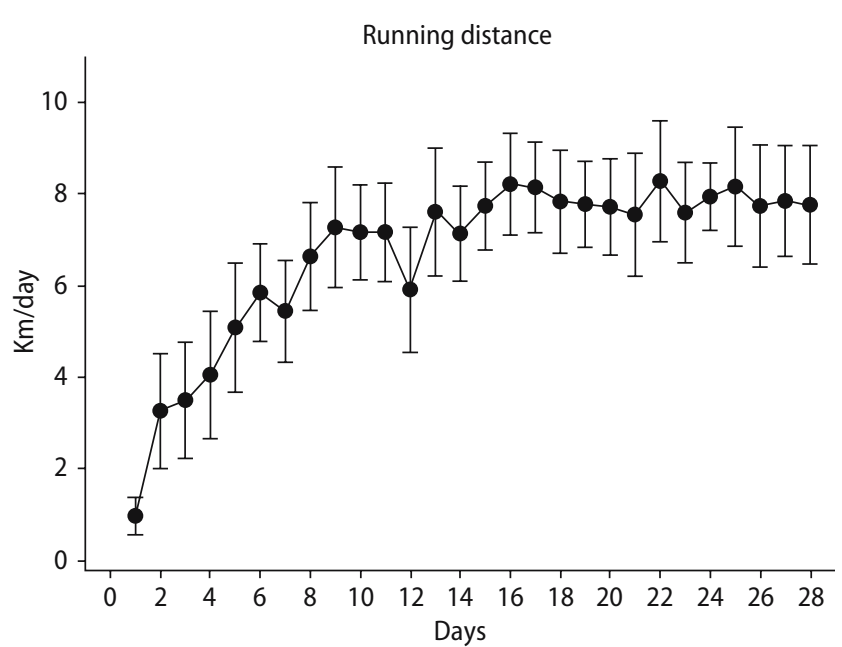

Fig. 1 The mean running distance of the mice during voluntary exercise $(n=9)$ was recorded in $\mathrm{km}$ per day. During the first 2 weeks, the distance run per day increased and reached a plateau until the end of the 4-week period

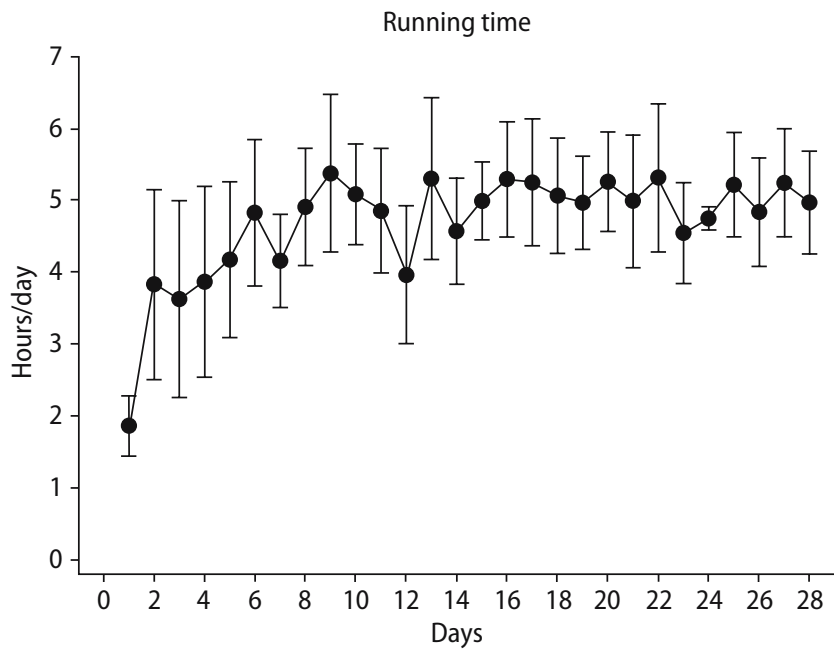

Fig. 2 The mean running time of the mice $(n=9)$ was recorded in hours per day. The increase in running time increased during the first days until a plateau was reached that was kept until the end of the 4-week period

Germany. All experiments described in this study comply with the current German laws and conform to the "Principle of laboratory animal care" (NIH publications 86-23, revised 1996).

\section{Preparation and tissue processing}

Mice were taken out of their cages and weighed (Table 1). After euthanization a median thoracotomy was performed and the hearts were immediately excised and fixed with $4 \%$ paraformaldehyde in PBS (pH 7.35, $1.395 \mathrm{osmol} / \mathrm{l})$. After storage of the whole hearts in the fixative for roughly $12 \mathrm{~h}$ the left ventricle including the interventricular septum was separated from the
Table 1 Animals: number per group, body, total heart and left ventricular mass

\begin{tabular}{llll}
\hline & CG & EG & $P$ \\
\hline Number of animals & 7 & 7 & - \\
$\mathrm{bm}(\mathrm{g})$ & $20.89(1.00)$ & $21.86(1.31)$ & 0.097 \\
$\mathrm{hm}(\mathrm{mg})$ & $135.57(13.23)$ & $160.29(31.19)$ & 0.209 \\
$\mathrm{hm} / \mathrm{bm}$ ratio $(\mathrm{mg} / \mathrm{g})$ & $6.49(0.52)$ & $7.30(1.11)$ & 0.097 \\
$\mathrm{lvm}(\mathrm{mg})$ & $93.44(9.54)$ & $102.61(16.88)$ & 0.535 \\
$\mathrm{lvm} / \mathrm{bm}$ ratio $(\mathrm{mg} / \mathrm{g})$ & $4.47(0.43)$ & $4.67(0.53)$ & 0.62 \\
$\mathrm{Ivv}\left(\mathrm{mm}^{3}\right)$ & $88.15(9.00)$ & $96.89(15.92)$ & 0.535 \\
\hline
\end{tabular}

Animals: Number per group, body, total heart and left ventricular mass. Abbreviations: $\mathrm{CG}=$ Control group; $\mathrm{EG}=$ Exercise group; $\mathrm{bm}=$ body mass; $\mathrm{hm}=$ heart mass; lvm = left ventricular mass; lvv = left ventricular myocardial volume

rest of the heart, weighed and cut along the longitudinal axis. The mass of the left ventricle was used to calculate the left ventricular volume by dividing the mass by the density of muscle tissue, $1.06 \mathrm{~g} / \mathrm{cm}^{3}[31]$. The two parts of the left ventricle were further cut three times transversally. From the eight tissue blocks four were chosen by systematic random sampling for paraffin embedding and estimation of the number of nuclei per myocyte. Each of these blocks was embedded in agar in isector moulds (rubber moulds with a spherical cavity with an average diameter of $5 \mathrm{~mm}$ ) to ensure isotropic orientation [34]. The isector-embedded tissue blocks were embedded in paraffin according to a standard protocol.

From the other four tissue blocks two were chosen randomly for electron microscopy. Since EM requires special fixation with glutaraldehyde, the specimens were incubated for $2 \mathrm{~h}$ in $1.5 \%$ glutaraldehyde, $1.5 \%$ paraformaldehyde in $0.15 \mathrm{M}$ HEPES buffer. Before further processing isotropic orientation was ensured by cutting the blocks according to the orientator principle [28]. The specimens were washed repeatedly in $0.15 \mathrm{M}$ HEPES buffer and $0.1 \mathrm{M}$ sodium cacodylate buffer, postfixed in $1 \%$ osmium tetroxide in $0.1 \mathrm{M}$ sodium cacodylate buffer, dehydrated in an ascending acetone series and finally embedded in araldite. All microscopic sections from the embedded tissues were obtained with calibrated microtomes/ultramicrotomes.

\section{Tissue shrinkage}

The processing of biological materials for microscopy may lead to tissue shrinkage and therefore, false estimations when numerical and volume densities are multiplied with the reference volume before embedding [11]. For this reason, two small tissue blocks were carefully weighed and, afterwards, embedded in araldite. The embedded samples were totally cut with a microtome advance of $2 \mu \mathrm{m}$ and every 25th section was mounted on a glass slide. Using the Cavalieri principle [14], the area of each of these sections was estimated, mul- 
Table 2 Summary of stereological parameters for estimation of cardiomyocyte and mitochondrial volume and number

\begin{tabular}{|c|c|c|c|}
\hline Parameter & Meaning & Method & Equation \\
\hline$V(s t r, r v)$ & $\begin{array}{l}\text { Total volume of a structure in } \\
\text { a given reference volume }\end{array}$ & Point counting & $V(\mathrm{str}, \mathrm{rv}):=\frac{\sum P(\mathrm{str})}{\sum P(\mathrm{rv})} \cdot V(\mathrm{rv})$ \\
\hline$N($ nuc,lv) & $\begin{array}{l}\text { Total number of nuclei in } \\
\text { the left ventricle }\end{array}$ & $\begin{array}{l}\text { Physical disector } \\
\text { (2 LM sections) }\end{array}$ & $N($ nuc, Iv $):=\frac{\sum Q^{-}(\text {nuc }) \cdot p}{2 \cdot h \cdot a \cdot \sum P(\mathrm{Iv})} \cdot V($ Iv $)$ \\
\hline $\bar{N}_{N}($ nuc $/$ fi $)$ & $\begin{array}{l}\text { Mean number of } \\
\text { nuclei per cardiomyocyte }\end{array}$ & $\begin{array}{l}\text { Physical disector } \\
\text { (14 LM sections) }\end{array}$ & $\begin{array}{l}\bar{N}_{N}(\text { nuc } / \text { fi }):= \\
\frac{\left.\sum\left[Q^{-}(\text {mono })+Q^{-}(\text {bi })+Q^{-}(\text {tri })+Q^{-}(\text {tetra })+Q^{-} \text {(penta }\right)\right]}{\sum Q^{-}(\text {mono })}+\frac{\sum Q^{-}(\text {bi })}{2}+\frac{\sum Q^{-}(\text {tri })}{3}+\frac{\sum Q^{-}(\text {tetra })}{4}+\frac{\sum Q^{-}(\text {penta })}{5}\end{array}$ \\
\hline$N(\mathrm{fi}, \mathrm{lv})$ & $\begin{array}{l}\text { Total number of cardiomyocytes } \\
\text { in the left ventricle }\end{array}$ & & $N(\mathrm{fi}$, Iv $):=\frac{N(\text { nuc }, \text { Iv })}{\bar{N}_{N}(\text { nuc } / \text { fi })}$ \\
\hline $\bar{V}_{N}(\mathrm{str})$ & Number-weighted mean volume & $\begin{array}{l}\text { Point counting } \\
\text { physical disector }\end{array}$ & $\bar{V}_{N}(\mathrm{str}):=\frac{V(\mathrm{str}, \mathrm{rv})}{N(\mathrm{str}, \mathrm{rv})}$ \\
\hline$N(\mathrm{mi}, \mathrm{lv})$ & $\begin{array}{l}\text { Total number of mitochondria } \\
\text { in the left ventricle }\end{array}$ & $\begin{array}{l}\text { Physical disector (2 EM sections) } \\
\text { Euler number }\end{array}$ & $N(\mathrm{mi}, \mathrm{Iv}):=\frac{\sum I-\sum B+\sum H}{2 \cdot h \cdot a} \times \frac{p}{\sum P(\mathrm{Iv})} \times V(\mathrm{Iv})$ \\
\hline
\end{tabular}

Summary of stereological parameters for estimation of cardiomyocyte and mitochondrial volume and number. Abbreviations: $V($ str,rv) $=$ Total volume of a structure within a reference volume; $P(\mathrm{str})=$ Points hitting a structure; $P(\mathrm{rv})=$ Points hitting the reference volume; $V(\mathrm{rv})=$ Reference volume; $N($ nuc,lv $)=$ Total number of cardiomyocyte nuclei in the left ventricle; $Q^{-}=$Number of nuclei counted in a set of disector pairs; $p=$ Number of points used for counting, if the four corners of the counting frame are used $P=4 ; h=$ Disector height; $a=$ Area of the unbiased counting frame; $P(\mathrm{lv})=$ Points hitting left ventricular myocardium; $V($ lv $)=V$ Volume of the left ventricle; $\bar{N}_{N}($ nuc $/$ fi $)=$ Mean number of nuclei per cardiomyocyte; $Q^{-}$(mono, bi, tri, tetra, penta) = Number of cardiomyocytes with one, two, three, four or five nuclei; $N(\mathrm{fi}, \mathrm{lv})=$ Total number of cardiomyocytes in the left ventricle; $\bar{V}_{N}(\mathrm{str})=$ Number-weighted volume of a structure; $N(\mathrm{mi}, \mathrm{lv})=$ Total number of mitochondria in the left ventricle; $I=$ Islands; $B=$ Bridges; $H=$ Holes

tiplied by the microtome advance of the section and by the total number of sections. This microscopically determined volume was compared to the original volume of the tissue blocks before embedding. In both cases, we observed a slight tissue shrinkage $\left(1.4 / 1.33 \mu \mathrm{m}^{3}=1.0526,3.9 / 3.8 \mu^{3}=1.0263\right)$. With an average volume shrinkage of $3.947 \%$, we decided not to include a correction factor.

\section{Estimation of numerical density of cardiomyocyte nuclei and volume density of myocardial compartments}

From the araldite tissue blocks serial sections were cut with a microtome advance of $1 \mu \mathrm{m}$ and physical disector pairs of $4-\mu \mathrm{m}$ disector height were collected and mounted on one glass slide and stained with methylene blue. At an objective lens magnification of $100 \times$ the volume densities of myocytes $\left(V_{V}(\mathrm{f} / \mathrm{ll})\right)$, myocyte nuclei $\left(V_{V}(\right.$ nuc/lv $\left.)\right)$ and interstitium $\left(V_{V}(\right.$ int/lv $\left.)\right)$ were estimated by point counting [39]. In this case, the interstitium comprised all left ventricular structures apart from cardiomyocytes. The total volumes of myocytes $(V(\mathrm{fi}, \mathrm{lv}))$, myocyte nuclei $(V($ nuc,lv $))$ and interstitium $(V($ int,lv) ) were obtained by multiplying the densities with the left ventricular volume.

At an objective magnification level of $20 \times$, the numerical density, $N_{\mathrm{V}}($ nuc/lv), and the total number of myocyte nuclei, $N($ nuc,lv), were estimated by the physical disector principle [36]. Whenever a myocyte nucleus appeared in the sampling section and was not observed in the reference section, it was counted. Afterwards, the test fields were used in the opposite direction, i.e. the former sampling section was used as the look-up section and vice versa. The numerical density of myocyte nuclei was calculated according to:

$N_{V}($ nuc $/ \mathrm{lv}):=\frac{\sum Q^{-}(\mathrm{nuc}) \cdot p}{2 \cdot h \cdot a \cdot \sum P(\mathrm{lv})}$

where $N_{V}$ (nuc/lv) is the number of nuclei per unit volume of the reference space, $Q^{-}$(nuc) is the number of myocyte nuclei counted, $p$ is the number of test points per counting frame (here 4$), \Sigma P(\mathrm{lv})$ is the number of points hitting myocardial tissue, $h$ is the disector height $(4 \mu \mathrm{m})$ and $a$ is the area of the unbiased counting frame. $\Sigma P(\mathrm{lv})$ is multiplied by 2 since the disector pairs were used for counting in both directions.

The total number of myocyte nuclei, $N$ (nuc,lv), was calculated by multiplying the numerical density, $N_{V}($ nuc $/ \mathrm{lv})$ with the total volume of the left ventricle (Table 2).

\section{Estimation of average number of nuclei per myocyte and total number of myocytes in the left ventricle}

From each paraffin block, 14 serial sections, $2.5 \mu \mathrm{m}$ in thickness, were generated and mounted on glass slides with 2 sections on each slide. According to Brüel and 
Nyengaard [8] the lateral borders of myocytes were visualised using a polyclonal rabbit antibody against collagen type IV (68124, ICN Biomedicals Inc., USA) and the intercalated discs as the end-to-end connection of myocytes were visualised using a monoclonal mouse antibody against cadherin (pan, C1821, Sigma-Aldrich, Denmark). Immunhistochemistry was performed as described in detail previously [8].

For estimation of the average number of nuclei per myocyte a modified Olympus BX-50 microscope with a motorized stage with capacity for 8 slides was used at an objective magnification of $20 \times$. The counting procedure is explained in detail in [8]. In short, the myocytes were sampled according to the physical disector principle based on their nuclei within local vertical windows on the 6th and 7th section. Following the sampled myocytes through all sections until they disappeared the number of nuclei within these myocytes was counted. The mean number of nuclei per cardiomyocyte was calculated and the total number of myocytes in the left ventricle was established by dividing the total number of myocyte nuclei by the mean number of nuclei per myocyte (Table 2 ).

\section{Estimation of mitochondrial volume density}

Mitochondrial volume density is closely correlated with the functional capacity of a muscle and can therefore be used as an indirect measure of the oxidative capacity of cardiomyocytes [40]. Ultrathin sections $(40-60 \mathrm{~nm})$ were cut from the araldite blocks, mounted on copper grids and stained with lead citrate and uranyl acetate. Using an EM 900 (Zeiss, Oberkochen, Germany), four sections per animal were investigated. Systematic uniform random test fields were superposed with a test grid with 18 points and 9 lines. The volume density of mitochondria $\left(V_{V}(\mathrm{mi} / \mathrm{fi})\right)$ was estimated by point counting [39] and converted to the total mitochondrial volume: $V(\mathrm{mi}, \mathrm{lv}):=V_{V}(\mathrm{mi} / \mathrm{fi}) \cdot V_{V}(\mathrm{~cm} / \mathrm{lv}) \cdot V(\mathrm{lv})$.

\section{Estimation of mitochondrial number and number-weighted mean volume of mitochondria}

Two adjacent ultrathin sections, $100 \mathrm{~nm}$ in thickness, were mounted on one copper grid and stained for EM. The two sections served as a physical disector with a height of $100 \mathrm{~nm}$. Systematic uniform random sampling was performed on these disector pairs at a primary magnification of $7,000 \times$. Digital images were taken from each pair of corresponding test fields. An unbiased counting frame with an area of $49 \mu \mathrm{m}^{2}$ was projected on each test field and corresponding test fields were compared with regard to mitochondrial connectivity. Whenever a mitochondrial profile was present in the sampling section and could not be detected in the reference section this was counted as an island. When two mitochondrial profiles were observed in the sampling section and were connected in the reference section, this was counted as a bridge. In very few cases, mitochondrial profiles showed a ring-like structure in the sampling section and a dense structure in the sampling section. This event was counted as a hole. From the counts of islands, bridges and holes, the Euler number $(\chi)$ was calculated according to the equation $\chi:=\sum I-\sum B+\sum H$ [24] where $I$ is the number of islands, $B$ the number of bridges and $H$ the number of holes. The Euler number was used to calculate the numerical density of mitochondria per unit volume of cardiomyocytes (Fig. 3 and Table 2).

From the numerical density of mitochondria the total number of mitochondria per cardiomyocyte and for the left ventricle were calculated by multiplying the numerical density with the corresponding reference volume. The number-weighted mean volume of mitochondria was obtained by dividing the total mitochondrial volume by the total number of mitochondria.

\section{Statistics}

All data are given as mean (standard deviation). Control and exercise animals were compared using the nonparametric 2-sided Whitney-Mann $U$-test for independent samples. Differences were considered statistically significant at $P<0.05$. Coefficients of variation are given in Table 3 . The total variation $\left(\mathrm{CV}_{\text {tot }}\right)$ is calculated as standard deviation divided by the mean. The coefficient of error $\left(\mathrm{CE}_{\text {met }}\right)$ is the error variance of the stereological methods. For the coefficient of error, the dominant noise effect was calculated. The CE for the number of nuclei and the number of nuclei per myocyte was estimated according to $\mathrm{CE}=1 / \sqrt{Q^{-}}$[24] and for the number of mitochondria according to $\mathrm{CE}=\frac{\sqrt{\sum B+\sum H+\sum I}}{\left|-\sum B+\sum H+\sum I\right|}[24]$. From the $\mathrm{CV}_{\text {tot }}$ and the $\mathrm{CE}_{\text {met }}$, the biological variation $\left(\mathrm{CV}_{\text {bio }}\right)$ can be determined by: $\mathrm{CV}_{\mathrm{bio}}=\sqrt{\mathrm{CV}_{\text {tot }}^{2}-\mathrm{CE}_{\text {met }}^{2}}$.

\section{Results}

\section{Heart and left ventricular mass}

The masses of the total hearts and of the left ventricles were similar in control and exercise mice. No differences were observed in the heart-to-body-mass ratio, the left ventricle-to-body-mass ratio and in left ven- 


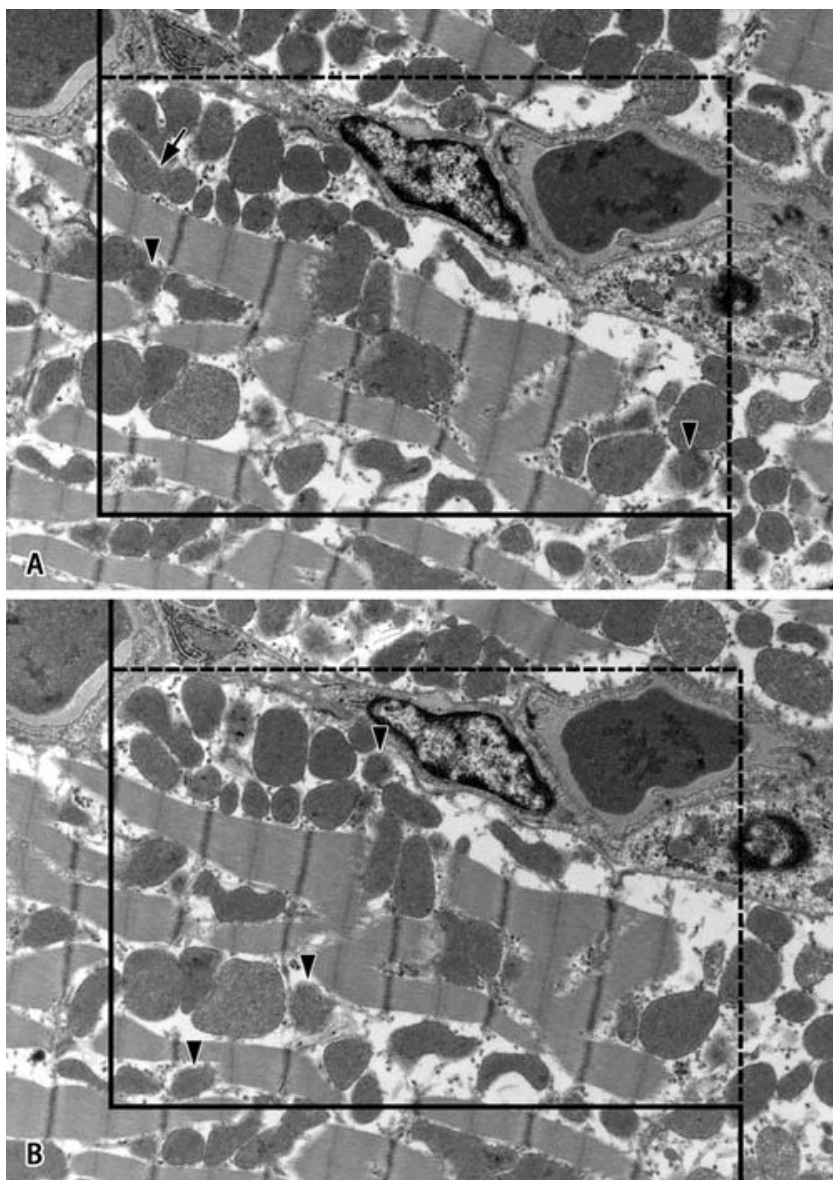

Fig. 3 Electron microscopic physical disector (A and B) for the estimation of mitochondrial number. The counting frame consists of a thick "exclusion" line and a dashed "inclusion" line and, for illustration reasons, has a larger area $\left(77 \mu \mathrm{m}^{2}\right)$ than the one used for counting in the present study. Counting events touching the exclusion line or its extensions are not counted whereas those touching the inclusion line are counted. The counting event "island" was defined through the presence of a mitochondrial profile (arrow heads) in the sampling section and its absence in the reference section. Please note that an island was also counted if there still was a "shadow" of the mitochondrion which - without the information of the sampling section-would not have been recognized as a mitochondrion. The counting event "bridge" was defined through the presence of two distinct mitochondrial profiles in the sampling section and a connection between them in the reference section (arrow). The counting event "hole" was relatively rare and is not shown in this figure. In this physical disector 5 islands and 1 bridge can be counted yielding an Euler number of $\chi=4$. Using the equation from Table 2, the numerical density of mitochondria referred to the cardiomyocyte as the reference volume can be estimated from this physical disector by $N_{\mathrm{V}}(\mathrm{mi} / \mathrm{fi}):=\frac{4}{2 \cdot 0.1 \mu \mathrm{m} \cdot 77 \mu \mathrm{m}^{2}} \cdot \frac{4}{3}=0.346 /$ $\mu \mathrm{m}^{3}$. Repeated estimations yielding a total Euler number of 100-200 lead to a sufficient precision of the estimate. Primary magnification: $7,100 \times$ )

tricular myocardial volume between the groups (Table 1).

\section{Cardiomyocyte number and volume}

The total number of cardiomyocytes in the left ventricle was comparably high in both groups and
Table 3 Estimates of coefficients of variation

\begin{tabular}{llll}
\hline & $\mathrm{CV}_{\text {tot }}$ & $\mathrm{CV}_{\text {bio }}$ & $\mathrm{CE}_{\text {met }}$ \\
\hline$N($ mi,lv $)$ & 0.107 & $*$ & 0.108 \\
$N($ nuc,lv) & 0.159 & 0.144 & 0.067 \\
$\bar{N}_{N}($ nuc $/$ fi $)$ & 0.062 & $*$ & 0.112
\end{tabular}

Estimates of coefficients of variation

Abbreviations: $N(\mathrm{mi}, \mathrm{lv})=$ Number of mitochondria in the left ventricle; $N($ nuc,lv $)=$ Number of nuclei in the left ventricle; $\bar{N}_{N}($ nuc $/ \mathrm{fi})=$ Mean number of nuclei per cardiomyocyte; $\mathrm{CV}_{\text {tot }}=$ Total coefficient of variation; $\mathrm{CV}_{\text {bio }}=$ Biological coefficient of variation; $\mathrm{CE}_{\mathrm{met}}=$ Coefficient of error. ${ }^{*}=$ The estimates of CE may vary quite a lot due to the limited amount of animals, therefore $\mathrm{CE}_{\text {met }}$ may sometimes be greater than $\mathrm{CV}_{\text {tot }}$, for which reason $\mathrm{CV}_{\text {bio }}$ cannot be calculated

Number of cardiomyocytes in the left ventricle
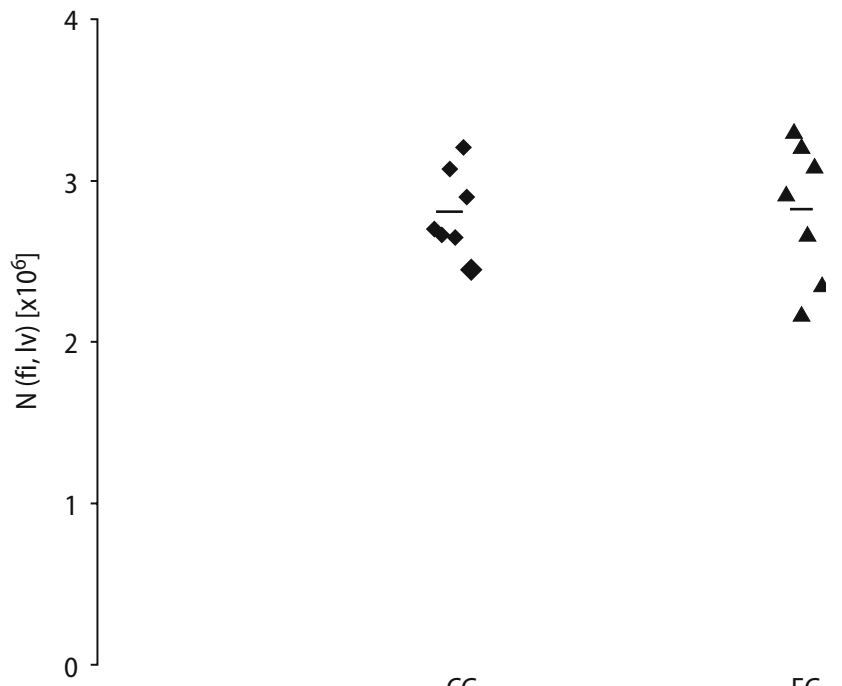

Fig. 4 The number of cardiomyocytes in the left ventricle was similar in both groups. $\mathrm{CG}=$ Control group. $\mathrm{EG}=$ Exercise group. The horizontal line indicates the mean of the individual values

ranged between 2.18 and 3.30 million (Fig. 4). No changes were observed in the number-weighted mean volume of cardiomyocytes between control and exercise animals (Fig. 5). A slight change in myocardial composition seemed to have occurred and was indicated by a tendency to a higher total volume of cardiomyocytes in EG $(P=0.097)$ (Table 4).

\section{Mitochondrial number and volume}

A highly significant increase in mitochondrial number (1.87 $\times$ ) was observed in the EG as compared to controls (Fig. 6). The total volume of mitochondria in the left ventricle was 1.48 times higher in EG than in CG whereas the number-weighted mean volume of 
Number-weighted mean volume of cardiomyocytes

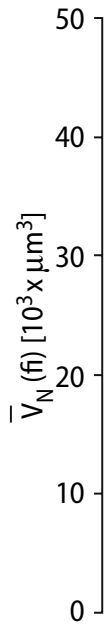

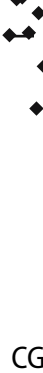

CG

EG

Fig. 5 The number-weighted mean volume of cardiomyocytes in the left ventricle was not changed by voluntary exercise. CG = Control group. $\mathrm{EG}=$ Exercise group. The horizontal line indicates the mean of the individual values

Table 4 Summary of stereological data

\begin{tabular}{|c|c|c|c|}
\hline & CG & EG & $P$ value \\
\hline$V_{v}(\mathrm{fi} / \mathrm{lv})(\%)$ & $77.8(6.3)$ & $84.4(4.5)$ & 0.073 \\
\hline$V_{V}($ nuc/lv) $(\%)$ & $2.0(0.8)$ & $2.0(0.6)$ & 0.902 \\
\hline$V_{V}$ (int/lv) (\%) & $20.3(6.7)$ & $13.8(4.4)$ & 0.128 \\
\hline$V(f i, l v)\left(\mathrm{mm}^{3}\right)$ & $68.7(10.0)$ & 81.6 (13.6) & 0.097 \\
\hline$V($ nuc,lv $)\left(\mathrm{mm}^{3}\right)$ & $1.8(0.8)$ & $2.0(0.9)$ & 0.710 \\
\hline$V\left(\right.$ int, lv) $\left(\mathrm{mm}^{3}\right)$ & $17.7(5.9)$ & $13.4(4.4)$ & 0.259 \\
\hline$N_{V}\left(\right.$ nuc/lv) $\left(10^{-5} / \mu \mathrm{m}^{3}\right)$ & $5.2(0.7)$ & $4.9(1.4)$ & 0.620 \\
\hline$N\left(\right.$ nuc, lv) $\left(10^{6}\right)$ & $4.5(0.5)$ & $4.6(0.7)$ & 0.710 \\
\hline $\bar{N}_{N}($ nuc $/ f i)$ & $1.62(0.08)$ & $1.62(0.11)$ & 1.000 \\
\hline$N(f i, l v)\left(10^{6}\right)$ & $2.81(0.27)$ & $2.82(0.43)$ & 0.710 \\
\hline $\bar{V}_{N}(\mathrm{fi})\left(10^{3} \times \mu \mathrm{m}^{3}\right)$ & $25.17(3.36)$ & $30.78(9.48)$ & 0.620 \\
\hline$V_{V}(\mathrm{mi} / \mathrm{fi})(\%)$ & $30.7(2.4)$ & $38.7(2.3)$ & 0.002 \\
\hline$V(\mathrm{mi}, \mathrm{Iv})\left(\mathrm{mm}^{3}\right)$ & $21.8(4.9)$ & $32.2(4.3)$ & 0.007 \\
\hline$N_{V}(\mathrm{mi} / \mathrm{fi})\left(1 / \mu \mathrm{m}^{3}\right)$ & $0.552(0.064)$ & $0.864(0.084)$ & $<0.001$ \\
\hline$N(\mathrm{mi}, \mathrm{lv})\left(10^{10}\right)$ & $3.759(0.441)$ & $7.015(1.130)$ & $<0.001$ \\
\hline $\bar{V}_{N}(\mathrm{mi})\left(\mu \mathrm{m}^{3}\right)$ & $0.581(0.117)$ & $0.46(0.05)$ & 0.017 \\
\hline$N(\mathrm{mi}, \mathrm{fi})$ & 13492 (1891) & $25886(8245)$ & $<0.001$ \\
\hline
\end{tabular}

Summary of stereological data. Abbreviations: $\quad C G=$ Control group; $\mathrm{EG}=$ Exercise group; $V_{V}=$ Volume density; $V=$ Total volume; $N_{V}=$ Numerical density; $N=$ Total number; $\bar{N}=$ Mean number; $\bar{V}_{N}=$ Number-weighted volume; $\mathrm{fi}=$ Cardiomyocyte; Iv = Left ventricle; nuc = Nuclei; int = Interstitium; $\mathrm{mi}=$ Mitochondria

mitochondria was higher in CG than in EG (1.26×) (Fig. 7).

\section{Discussion}

The present study is the first to investigate the quantitative structural changes of volume and number of cardiomyocytes and their mitochondria in the
Number of mitochondria per cardiomyocyte

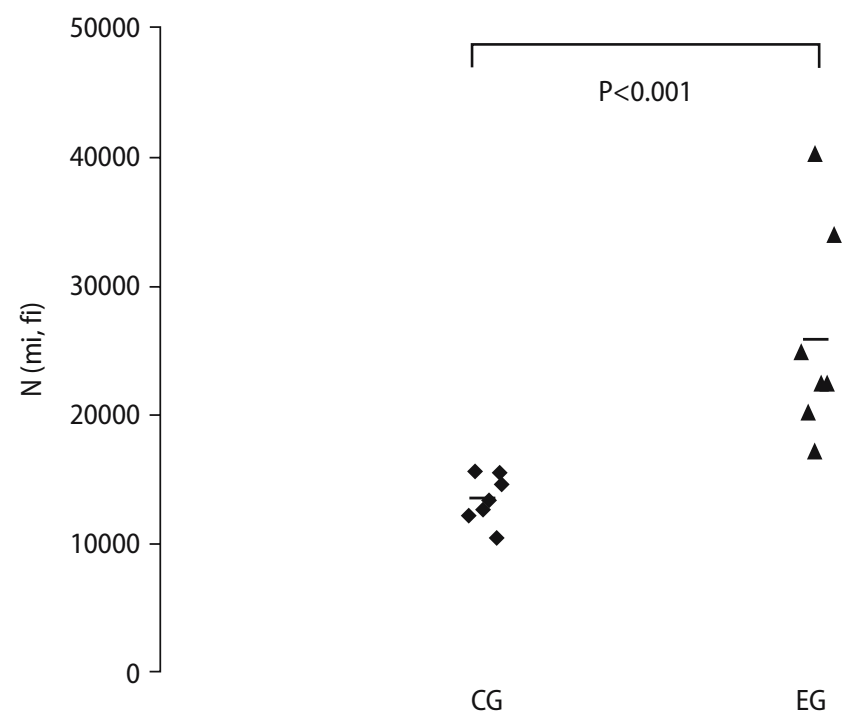

Fig. 6 The number of mitochondria per cardiomyocyte was significantly higher in the exercise (EG) than in the control group (CG). The horizontal line indicates the mean of the individual values

Number-weighted mean volume of mitochondria
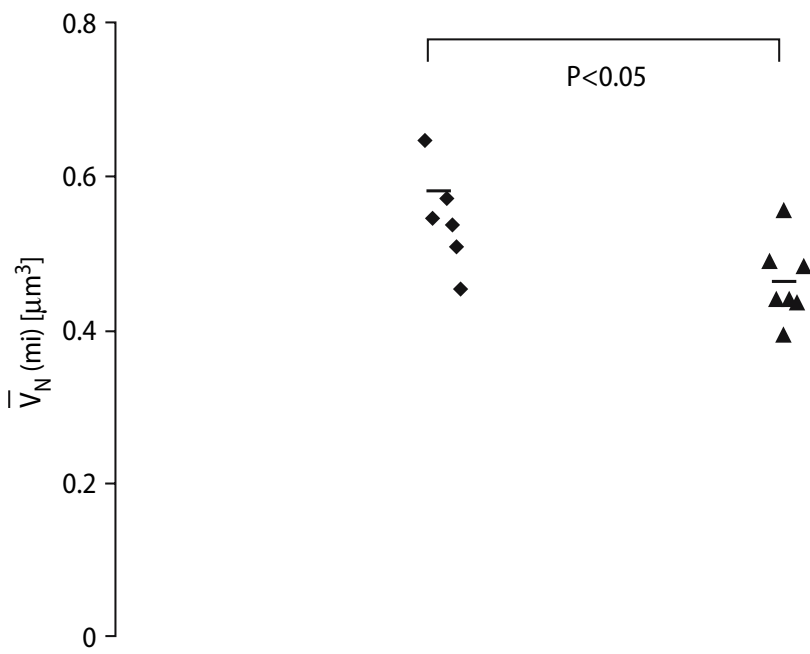

CG

EG

Fig. 7 The number-weighted mean volume of cardiomyocyte mitochondria was significantly lower in EG (exercise group) than in CG (control group). The horizontal line indicates the mean of the individual values

left ventricle of mice after a period of voluntary wheel running. Four weeks of VE did not induce changes in total heart and left ventricular mass nor in cardiomyocyte mean volume or number. The subcellular composition of cardiomyocytes was characterized by an increase in total mitochondrial volume as well as 
an increased number of mitochondria in exercised mice compared to sedentary controls. From a methodological point of view the present study employs state-of-the-art stereology that gives estimates of total numbers, volumes and number-weighted mean volumes of cardiomyocytes and mitochondria.

Myocardial hypertrophy occurs physiologically during postnatal development $[2,29,42]$ and as an adaptation to an increased workload during exercise [26]. Hypertrophy is usually referred to as a growth mechanism characterized by increasing cell size, which is to be differentiated from hyperplasia, growth by increasing cell number. According to the concept of cardiomyocytes being terminally differentiated cells [25], exercise induces myocardial growth by a hypertrophic mechanism. Parameters that characterize myocardial hypertrophy are therefore increased mass or volume of the heart and its chambers, increased total cardiomyocyte and/or interstitial volumes, increased mean cardiomyocyte volume and normal total cardiomyocyte number. A useful parameter may also be the mean myocyte volume per nucleus if interpreted carefully [29]. In our animal model, 4 weeks of VE did not lead to an increased left ventricular mass compared to the sedentary controls. This finding is in accordance with other studies on VE [30] but also contrasts with some reports $[1,22]$. In a stereological study on the effects of exercise and inactivation in the woodmouse it was reported that inactivation did not induce changes in body or heart mass but exercise rather reduced the mass of the heart [17]. These controversial observations may be influenced by the duration of exercise, food composition, gender and species [23]. In our study, the mode and duration of exercise per day was comparable to or even higher than that in other studies. It therefore seems unlikely that the degree of exercise was too low in the present study. Since it is difficult to control the exercise conditions and the running distance and duration values are cumulative data per day, it cannot be ruled out completely that there are differences in the running characteristics of our study and those of others, which may affect the cardiac response mechanisms. We used female mice because it is known that exercise-induced cardiac hypertrophy occurs more frequently in female than in male rodents [23]. However, mice were fed standard food, which contained phytoestrogens. In a recent study, Gardner et al. [13] showed that dietary phytoestrogens inhibit ventricular remodelling due to volume overload in female rats. It appears reasonable that this explains why no changes in heart mass occurred as a consequence of running.

In accordance with the unchanged left ventricular volume, we did not find changes in cardiomyocyte volume or number. The total number of cardiomyo- cytes in the left ventricle of mice hearts was estimated to be between 2.18 and 3.30 million. These numbers are approximately 10 times smaller than those reported for the left ventricle of the rat heart [8] which is in good accordance with the differences in body size between mouse and rat. Looking at the volume densities of cardiomyocytes and interstitium they are in good accordance with previous reports [27].

Controversial reports also exist about the changes in subcellular compartments of cardiomyocytes, however, all of these studies were performed using a given forced exercise schedule (swimming or treadmill running) instead of VE. Several studies have reported an increase in the mitochondria-to-myofibril ratio reflected by increasing mitochondrial and decreasing myofibril volume fractions $[6,9]$. Other authors did not find evidence that the subcellular composition of cardiomyocytes changes as a consequence of endurance training [3, 27]. While these differences among the studies may be caused by different experimental protocols (cf. above) it needs to be stressed that they may also arise from the use of different morphometric methods and that the interpretation of ratios can easily lead to misinterpretations. Unbiased stereological estimates of total numbers and volumes should therefore be preferred to ratios whenever possible which is the case in most experimental studies.

The present study provides evidence that the total volume of mitochondria increases significantly in the course of VE, which increases the oxidative capacity of the heart $[18,41]$. To clarify the mechanisms by which this volume increase occurs we estimated the total number of mitochondria and their number-weighted mean volume by applying the Euler-Poincare characteristic [24]. We observed that there is a highly significant increase in mitochondrial number but not in the mean volume of the individual mitochondria. In fact, the mean mitochondrial size was even smaller in EG than in CG. This result implies that the heart rather builds new mitochondria of the same size than increasing the size of the existing mitochondria. The biological reason for preferring this mechanism may lie in the aim of keeping diffusion distances for oxygen and ATP as small and diffusion area as large as possible. An increase in oxidative capacity of the heart is in good accordance with the increased maximal oxygen uptake described by others [19]. We also detected an increase in the volume fraction of mitochondria, a parameter often used by other authors. This increase in mitochondrial volume density correlates with the findings of Hoppeler et al. [17] on inactivated and exercised woodmice but contrasts with the reports of Anversa et al. [3], Mattfeldt et al. [27] and Kayar et al. [21] who did not observe changes in mitochondrial volume density due to exercise. However, as a consequence of 
exercise these authors found an increase in heart mass, which was not observed in our study. An increase in heart mass combined with a constant mitochondrial volume fraction, however, means that the total volume of mitochondria has also increased in their studies. It seems reasonable to conclude that an increase in mitochondrial volume is the first structural adaptation to endurance training and leads to an increase in volume density, total volume and number of mitochondria and a corresponding decrease in myofibrillar volume fraction. With a more pronounced training schedule, the volume of myofibrils increases which reverses the increase in mitochondrial volume fraction despite the elevated total mitochondrial volume and number.

In summary, it is reasonable to conclude that the effects of VE do not go along with an enlargement of the whole heart but by subcellular changes that increase the myocardial oxidative capacity. These alterations are caused by a higher number and total volume of mitochondria and adapt the heart to a higher metabolic demand. Further studies need to compare these results to strenuous forms of exercise and pressure-induced hypertrophy. The stereological methodology presented within this paper is ideally suited to solve those problems in cardiac research, which demand a distinction between the growth by increasing number or size of cardiomyocytes or subcellular organelles. It is also relevant whenever a distinction needs to be made between a significant cell loss, be it apoptosis or necrosis, and the dysfunction of the existing cells caused, for example, by a misrelationship between metabolic demands and oxidative capacity.

Acknowledgments The authors wish to thank Ms. S. Freese, Ms. H. Hühn, Ms. S. Kosin, Ms. R. Waldmann-Beushausen, Ms. S. Wienstroth (all Göttingen), Ms. A. Berg and Ms. M.-B. Lundorf (both Aarhus) for their expert technical assistance.

\section{References}

1. Allen DL, Harrison BC, Maass A, Bell ML, Byrnes WC, Leinwand LA (2001) Cardiac and skeletal muscle adaptations to voluntary wheel running in the mouse. J Appl Physiol 90:1900-1908

2. Anversa P, Olivetti G, Loud AV (1980) Morphometric study of early postnatal development in the left and right ventricular myocardium of the rat. Circ Res 46:495-502

3. Anversa P, Beghi C, Levicky V, McDonald SL, Kikkawa Y (1982) Morphometry of right ventricular hypertrophy induced by strenuous exercise in rat. Am J Physiol 243:H856-H861

4. Anversa P, Beghi C, McDonald SL, Levicky V, Kikkawa Y, Olivetti G (1984) Morphometry of right ventricular hypertrophy induced by myocardial infarction in the rat. Am J Pathol 116:504-513

5. Arcos JC, Sohal RS, Sun SC, Argus MF, Burch GE (1968) Changes in ultrastructure and respiratory control in mitochondria of rat heart hypertrophied by exercise. Exp Mol Pathol 8:49-65

6. Bozner A, Meessen H (1969) The ultrastructure of the myocardium of the rat after single and repeated swim exercises. Virchows Arch B Cell Pathol 3:248-269

7. Brændgaard H, Gundersen HJ (1986) The impact of recent stereological advances on quantitative studies of the nervous system. J Neurosci Meth 18:39-78
8. Brüel A, Nyengaard JR (2005) Designbased stereological estimation of the total number of cardiac myocytes in histological sections. Basic Res Cardiol 100:311-319

9. Crisman RP, Tomanek RJ (1985) Exercise training modifies myocardial mitochondria and myofibril growth in spontaneously hypertensive rats. Am J Physiol 248:H8-H14

10. Deussen A, Brand M, Pexa A, Weichsel J (2006) Metabolic coronary flow regulation-current concepts. Basic Res Cardiol 101:453-464

11. Dorph-Petersen KA, Nyengaard JR, Gundersen HJ (2001) Tissue shrinkage and unbiased stereological estimation of particle number and size. J Microsc 204:232-246

12. Fagard R (2003) Athlete's heart. Heart 89:1455-1461

13. Gardner JD, Brower GL, Janicki JS (2005) Effects of dietary phytoestrogens on cardiac remodeling secondary to chronic volume overload in female rats. J Appl Physiol 99:1378-1383

14. Gundersen HJ, Jensen EB (1987) The efficiency of systematic sampling in stereology and its prediction. J Microsc 147:229-263

15. Holloszy JO (1997) Exercise increases average longevity of female rats despite increased food intake and no growth retardation. J Gerontol A Biol Sci Med Sci 48:B97-B100
16. Holloszy JO (1998) Mortality rate and longevity of food-restricted exercising male rats: a reevaluation. J Appl Physiol 82:399-403

17. Hoppeler H, Lindstedt SL, Uhlmann A, Niesel A, Cruz-Orive LM, Weibel ER (1984) Oxygen consumption and the composition of skeletal muscle tissue after training and inactivation in the European woodmouse (Apodemus sylvaticus). J Comp Physiol B 155:51-61

18. Hoppeler H, Kayar SR, Claassen H, Uhlmann E, Karas RH (1987) Adaptive variation in the mammalian respiratory system in relation to energetic demand: III. Skeletal muscles: setting the demand for oxygen. Respir Physiol 69:2746

19. Jones AM, Carter H (2000) The effect of endurance training on parameters of aerobic fitness. Sports Med 29:373-386

20. Judge S, Jang YM, Smith A, Selman C, Phillips T, Speakman JR, Hagen T, Leeuwenburgh C (2005) Exercise by lifelong voluntary wheel running reduces subsarcolemmal and interfibrillar mitochondrial hydrogen peroxide production in the heart. Am J Physiol Regul Integr Comp Physiol 289:R1564R1572

21. Kayar SR, Conley KE, Claassen H, Hoppeler H (1986) Capillarity and mitochondrial distribution in rat myocardium following exercise training. J Exp Biol 120:189-199 
22. Kingwell BA, Arnold PJ, Jennings GL, Dart AM (1998) The effects of voluntary running on cardiac mass and aortic compliance in Wistar-Kyoto and spontaneously hypertensive rats. J Hypertens 16:181-185

23. Konhilas JP, Mass AH, Luckey SW, Stauffler BL, Olson EN, Leinwand LA (2004) Sex modifies exercise and cardiac adaptation in mice. Am J Physiol Heart Circ Physiol 287:H2768-H2776

24. Kroustrup JP, Gundersen HJ (2001) Estimating the number of complex particles using the ConnEulor principle. J Microsc 203:314-320

25. MacLellan WR, Schneider MD (2000) Genetic dissection of cardiac growth control pathways. Annu Rev Physiol 62:289-319

26. Maron BJ, Pelliccia A (2006) The heart of trained athletes. Cardiac remodelling and the risks of sports, including sudden death. Circulation 114:1633-1644

27. Mattfeldt T, Krämer K-L, Zeitz R, Mall G (1986) Stereology of myocardial hypertrophy induced by physical exercise. Virchows Arch [Pathol Anat] 409:473-484

28. Mattfeldt T, Mall G, Gharehbaghi H, Moller P (1990) Estimation of surface area and length with the orientator. J Microsc 159:301-317
29. Mayhew TM, Pharaoh A, Austin A, Fagan DG (1997) Stereological estimates of nuclear number in human ventricular cardiomyocytes before and after birth obtained using physical disectors. J Anat 191:107-115

30. Mayhew TM, Huppertz B, Kaufmann P, Kingdom JC (2003) The 'reference trap' revisited: examples of the dangers in using ratios to describe fetoplacental angiogenesis and trophoblast turnover. Placenta 24:1-7

31. Mendez J, Keys A (1961) Density and composition of mammalian muscle. Metabolism 9:184-188

32. Natali AJ, Turner DL, Harrison SM, White E (2001) Regional effects of voluntary exercise on cell size and contraction-frequency responses in rat cardiac myocytes. J Exp Biol 204:11911199

33. Noakes TD (2006) The limits of endurance exercise. Basic Res Cardiol 101:408-417

34. Nyengaard JR, Gundersen HJ (1992) The isector: a simple and direct method for generating isotropic, uniform random sections from small specimens. J Microsc 165:427-431

35. Reichmann H, Hoppeler H, MathieuCostello $\mathrm{O}$, von Bergen F, Pette D (1985) Biochemical and ultrastructural changes of skeletal muscle mitochondria after chronic electrical stimulation in rabbits. Pflügers Arch 404:1-9
36. Sterio DC (1984) The unbiased estimation of number and sizes of arbitrary particles using the disector. J Microsc 134:127-136

37. Szibor M, Holtz J (2003) Mitochondrial ageing. Basic Res Cardiol 98:210-218

38. Vanoli E, Cerati D, Pedretti RFE (1998) Autonomic control of heart rate: pharmacological and nonpharmacological modulation. Basic Res Cardiol 93:133-142

39. Weibel ER (1979) Stereological methods. Vol. 1: Practical methods for biological morphometry. Academic Press, London

40. Weibel ER (2000) Symmorphosis. On form and function in shaping life. Harvard University Press, Cambridge

41. Weibel ER, Bacigalupe LD, Schmitt B, Hoppeler H (2004) Allometric scaling of maximal metabolic rate in mammals: muscle aerobic capacity as determinant factor. Respir Physiol Neurobiol 140:115-132

42. Wulfsohn D, Nyengaard JR, Tang Y (2004) Postnatal growth of cardiomyocytes in the left ventricle of the rat. Anat Rec A Discov Mol Cell Evol Biol 277:236-247 